\title{
The growth and properties of self-assembled GaN nanorods, nanoparticles and nanotubes on Si substrates by annealing GaN/ZnO films
}

\author{
Yuping Cao ${ }^{1, \mathrm{a}}$, Tao $\mathrm{Hu}^{1, \mathrm{~b}}$ \\ ${ }^{1}$ Institute of science, Qilu University Of Technology, Jinan, Shandong, 250353, China \\ ashshcyp@163.com, btaotao_phy@qq.com
}

\section{Keywords: GaN; nanostructures; sputtering}

Abstract. GaN nanostructured films have been successfully grown through radio frequency magnetron sputtering system via annealing $\mathrm{GaN} / \mathrm{ZnO}$ films in ammonia ambient at $950^{\circ} \mathrm{C}$. The volatilization of $\mathrm{ZnO}$ buffer layer plays an important role in the formation process of $\mathrm{GaN}$ nanostructures. X-ray diffraction (XRD), transmission electron microscope (TEM), x-ray photoelectron spectroscopy (XPS) and photoluminescence (PL) are used to analyze the structure, morphology, composition and optical properties of the as-grown products. The XRD and selected area electron diffraction (SAED) indicate that the reflections of the samples can be indexed to the hexagonal GaN phase. TEM shows that the products are of pure hexagonal GaN single crystal. By XPS spectra, quantification of peaks gives the atomic ratio of $\mathrm{Ga}$ to $\mathrm{N}$ of about 1:1.1. The photoluminescence (PL) spectrum indicates that the GaN have a good emission property, which will have potential advantages for applications in laser device using one-dimensional structures. Finally, the proposed growth mechanism is also briefly discussed.

\section{Introduction}

One-dimensional (1D) nanoscale GaN materials, such as nanotubes, nanowires and nanobelts, have aroused intensive research interest due to their importance for fundamental studies of size-dependent physical and chemical properties and for their potential application in nanodevices [1-3]. The promise of UV photoconducting devices for optical/electronic nanoscale switching devices has been fulfilled [4]. Such devices would be wonderful test beds for studying quantum effects in one-dimensional structures. On the other hand, the growth of larger diameter rods could be very useful for optical devices and electronic applications [5]. Until now, there have been many reports on the formation of one-dimensional GaN structures using various methods by several groups [6-7]. However, these methods for the synthesis of GaN nanostructures are chemical technique, for example, the reactions between $\mathrm{Ga}$ or $\mathrm{Ga}_{2} \mathrm{O}_{3}$ films and $\mathrm{NH}_{3}$. In this paper, we report on an effective method to form the $\mathrm{GaN}$ nanorods, nanoparticles and nanotubes on Si substrates by annealing the GaN/ZnO films. We use the volatilization of $\mathrm{ZnO}$ layer for the growth of $\mathrm{GaN}$ nanostructures. This growth method is applicable to continuous synthesis and able to produce a large number of single-crystalline GaN nanostructures with a relatively high purity and at a low cost.

\section{Experimental details}

In our experiment, the growth process of the GaN nanoscale films could be devided into two steps. The first step was that $\mathrm{ZnO}$ thin films were deposited on Si substrates by sputtering $\mathrm{ZnO}$ target (99.999\%) in a JCK-500A radio frequency magnetron sputtering system. The sputtering condition was as follows: a background pressure was $4.0 \times 10^{-4} \mathrm{~Pa}$; the distance between the target and the substrates was $80 \mathrm{~mm}$; the pressure of Ar gas (99.999\%) was $2 \mathrm{~Pa}$; the output voltage of steady current device was $200 \mathrm{~V}$ and the output current was $220 \mathrm{~mA}$. After $15 \mathrm{~min}$ sputtering, $\mathrm{ZnO}$ films with the thickness of about $300 \mathrm{~nm}$ were obtained. The as-grown $\mathrm{ZnO}$ thin films were lately annealed in oxygen ambient at the temperature of $700^{\circ} \mathrm{C}$. The second step was that $\mathrm{GaN}$ thin films were deposited on $\mathrm{ZnO} / \mathrm{Si}(111)$ substrates by sputtering $\mathrm{GaN}$ target $(99.999 \%)$ in the same sputtering 
system. The different sputtering condition was that: a base pressure was $3.0 \times 10^{-4} \mathrm{~Pa}$; the work pressure of Ar gas (99.999\%) was $3 \mathrm{~Pa}$; the radio frequency generator was set to $150 \mathrm{~W}$ and the frequency was $13.56 \mathrm{MHz}$; the sputtering time was 90 min and the thickness of GaN film was about $500 \mathrm{~nm}$. The obtained GaN films were amorphous as deposited and needed to be annealed. Subsequently, the quartz boat with the samples was placed into the constant temperature region for annealing. Above all, the flowing N2 was introduced into the tube to flush out of the residual air for 5 $\mathrm{min}$, and then ammonia was flowed into the tube with a flow rate of $500 \mathrm{~mL} / \mathrm{min}$ for $15 \mathrm{~min}$ at $950^{\circ} \mathrm{C}$ while $\mathrm{N}_{2}$ was switched off. After annealed, the samples were cooled down to room temperature in the $\mathrm{N}_{2}$ ambient.

A Rigaku D/max-rB X-ray diffraction (XRD) meter with $\mathrm{Cu}$ Ka-line, Hitachi H-800 transmission electron microscope (TEM), Philips TECNAI-20 high-resolution transmission electron microscopy (HRTEM), MICROLAB MK II X-ray photoelectron spectroscopy (XPS) and LS50-B fluorescence spectrophotometer were applied to examine the structure, morphology, composition and photoluminescence properties of the GaN nanostructures.

\section{Results and discussion}

XPS analysis. Fig. 1 shows the XPS for the GaN synthesized at the annealing temperature of $950^{\circ} \mathrm{C}$. It is obvious in Fig. 1(a) that the peak of Ga3d locates at the binding energy of $20.0 \mathrm{eV}$. According to the report [8], the peak at $19.7 \mathrm{eV}$ corresponds to $\mathrm{GaN}$, indicating that the $\mathrm{Ga}$ atoms in the film are in the compound state $(\mathrm{GaN}$, not the elemental $\mathrm{Ga} 18.4 \mathrm{eV})$. The core lever of $\mathrm{Ga}$ has a positive shift from elemental $\mathrm{Ga}$. This shift in binding energies of $\mathrm{Ga}$ and $\mathrm{N}$ confirms that the bonding between $\mathrm{Ga}$ and $\mathrm{N}$ as well as the absence of elemental gallium. The peak of N1s is observed at 397.5 $\mathrm{eV}$ in Fig. 1(b), which is consistent with the presence of chemisorbed nitrogen along with nitrogen in $\mathrm{GaN}$ [9]. Quantification of peaks gives the atomic ratio of $\mathrm{Ga}$ to $\mathrm{N}$ of 1:1.1. Since XPS analysis is performed for the GaN film exposed to air, O1s and C1s core level (not shown) peaks are always detected besides Ga3d and N1s. The O1s peak centered at $531.0 \mathrm{eV}$ is given in Fig.1(c). According to Amanullah's report [10], generally, the O1s peak has been observed in the binding energy region of $529-535 \mathrm{eV}$. The peak around $529-530 \mathrm{eV}$ is ascribed to lattice oxygen. For chemisorbed $\mathrm{O}_{2}$ on the surface the binding energy are ranged from $530.0 \mathrm{eV}$ to $530.9 \mathrm{eV}$. Therefore, the $\mathrm{O} 1 \mathrm{~s}$ peak in the present work is attributed to chemisorbed oxygen.
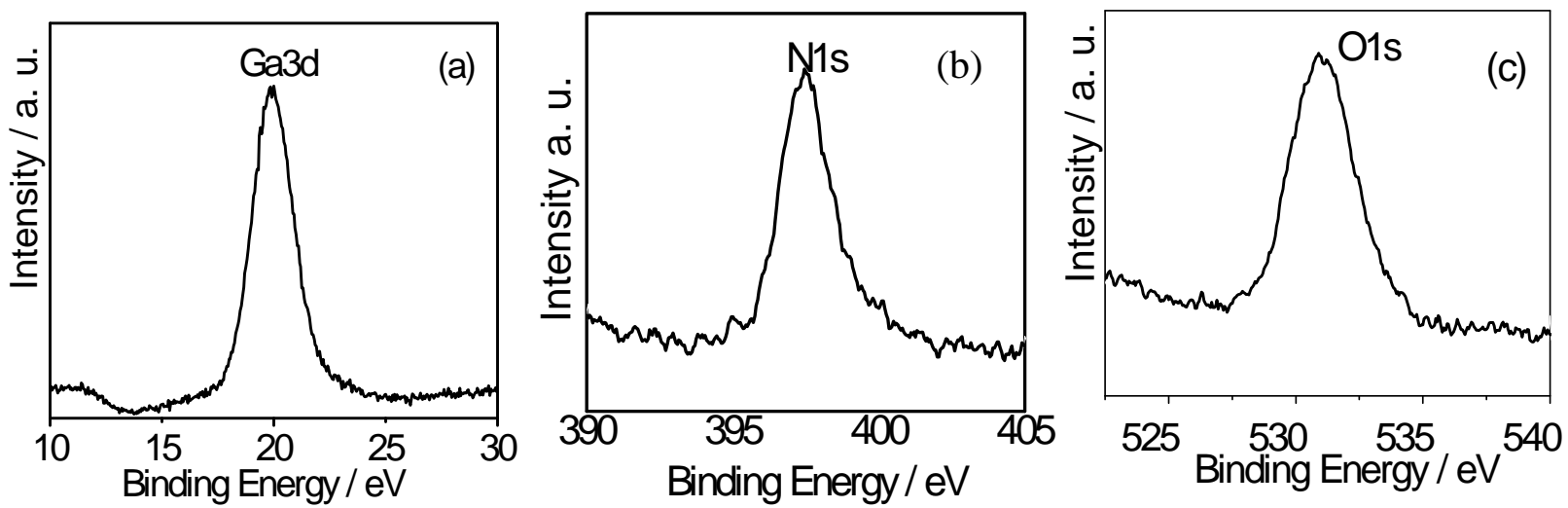

Fig. 1 XPS spectra obtained from the samples annealed at $950{ }^{\circ} \mathrm{C}$.

TEM analysis. Fig. 2(a) shows the TEM morphology of a cluster of the GaN nanorods, which are as long as several-hundred nanometers with an average diameter ranging from $80 \mathrm{~nm}$ to $200 \mathrm{~nm}$. Fig. 2(b) presents the HRTEM image of a single nanorod, which is as long as $350 \mathrm{~nm}$ with the diameter of about $120 \mathrm{~nm}$. The HRTEM lattice image of the single nanorod is illustrated in Fig.2(c). The clear lattice fringes further confirm that the synthesized nanorods are of single-crystal GaN. The interplanar spacing is about $0.260 \mathrm{~nm}$, which is close to the value of the $\{002\}$ plane spacing of hexagonal GaN. The single-crystal GaN nanorods can also be identified from the SAED pattern shown in the inset of Fig. 2(c), which is ascribed to the reflection of hexagonal wurtzite GaN single crystal. Fig. 2(d) and 
fig. 2(e) show the TEM morphology and the corresponding selected area electron diffraction (SAED) pattern of the nanoparticles, which also confirms the $\mathrm{GaN}$ has a hexagonal wurtzite structure. Also, some nanotubes are illustrated on the TEM with the inner diameter of about $25 \mathrm{~nm}$ and outer diameter of $41 \mathrm{~nm}$ as shown in Fig.2(f), but these GaN nanotubes are amorphous, which have not become crystal and need a further growth.

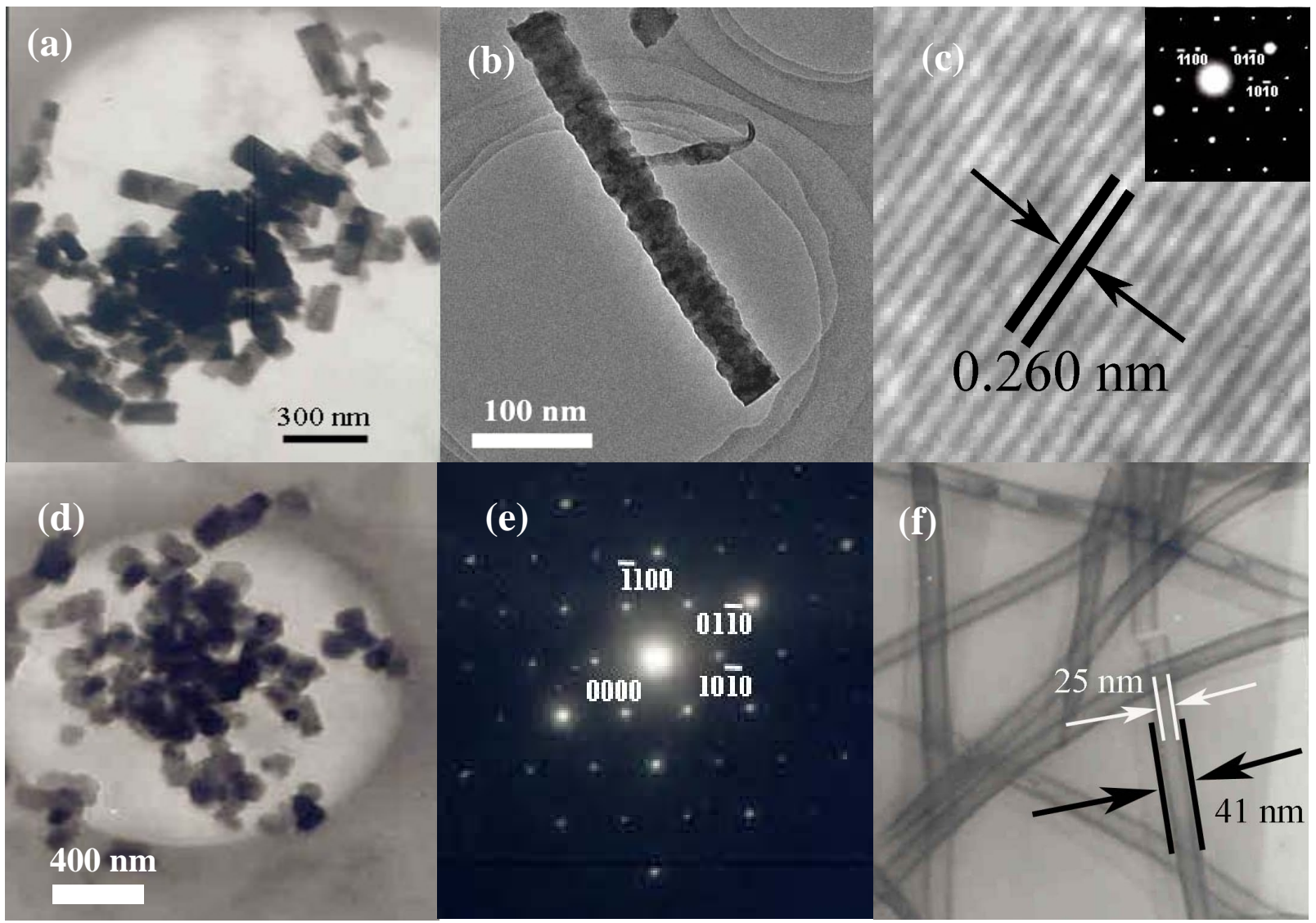

Fig. 2 TEM morphology, lattice image and the corresponding selected area electron diffraction pattern of the synthesized GaN nanostructured films.

XRD analysis. Fig. 3 shows the XRD pattern of the GaN samples annealed at $950^{\circ} \mathrm{C}$. Three peaks located at $32.36^{\circ}, 34.54^{\circ}$ and $36.78^{\circ}$ can be indexed to (100), (002), and (101) of GaN, demonstrating that the reflections correspond to the hexagonal GaN wurtzite structure with the lattice constants of $a=0.318 \mathrm{~nm}$ and $c=0.518 \mathrm{~nm}$, which are in good agreement with the already reported GaN values [11]. The spectrum has a preferred (002) orientation. This c-axis orientation is believed to result from the lowest surface energy of the (002) basal plane in GaN, leading to a preferred growth in the [002] direction. The sharp diffraction peaks also reveal that the GaN nanostructures thus synthesized have a high quality.

PL analysis. Fig. 4 shows the PL spectrum of as-grown GaN nanostructured films. The dominant peak centered at $374.1 \mathrm{~nm}$ corresponds to the near-band-edge emission in a wurtzite $\mathrm{GaN}$ epitaxial layer [12]. Because the as-grown GaN nanorods are too large for quantum confinement effects, and the diameter of the thinnest nanorod is even much larger than the Bohr exciton radius $(11 \mathrm{~nm})$ of GaN, the UV light emission has no blue shift from the band-gap emission compared with bulk GaN [12]. From the PL spectra, we can also see that the peak has a slightly red shift. In the reference [13], this shift is ascribed to donor-acceptor transition revealing high donor and acceptor concentrations due to extrinsic or intrinsic defects. A recent work on surface damage in $\mathrm{GaN}$ [14] attributes surface damage to a $374.1 \mathrm{~nm}$ peak. As $\mathrm{GaN}$ films are formed with $\mathrm{ZnO}$ buffer layers, $\mathrm{Zn}$ may be diffused in $\mathrm{GaN}$ in the process of the ammoniation [15]. So the emission peak at $435.9 \mathrm{~nm}$ may correspond to the transition of $\mathrm{Zn}$ impurity energy level. It may be also ascribed to the electron transition from the deep 
acceptor level, resulting from $\mathrm{C}$ impurity taking place of $\mathrm{N}$ atoms, to the conductor band [16]. In other references [17], another peak at $474.8 \mathrm{~nm}$ may be ascribed to existence of defects or surface states. The commonly observed yellow luminescence band centered at $2.2-2.3 \mathrm{eV}$ is not detected, indicating that our nanorods are of stoichiometric composition and possess a high optical quality. However, further work is needed to investigate the PL mechanism of the GaN nanostructured films.

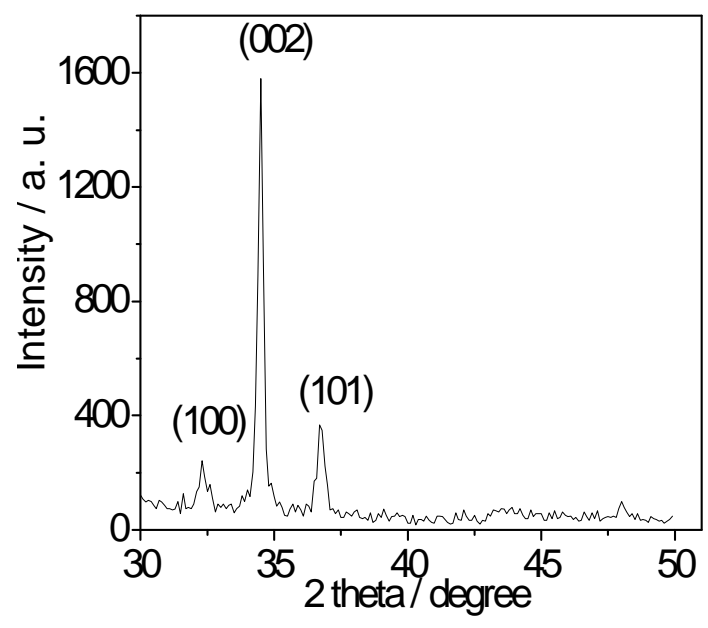

Fig. 3 XRD pattern of the $\mathrm{GaN}$ nanostructures annealed at $950^{\circ} \mathrm{C}$.

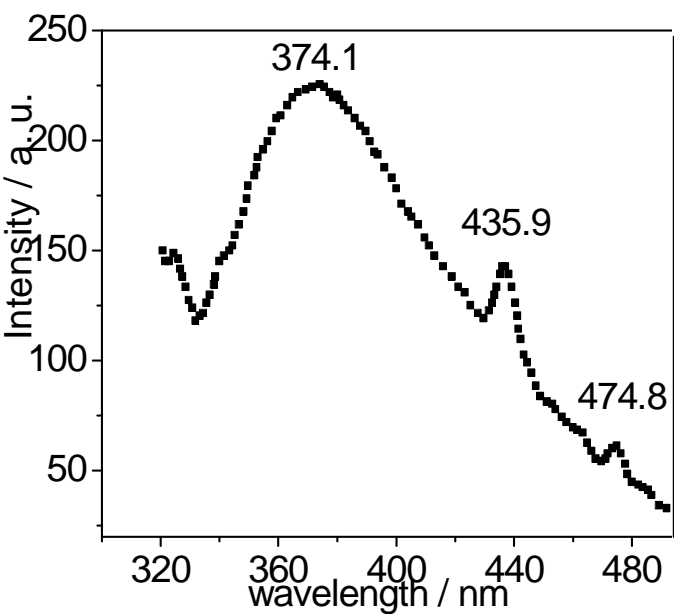

Fig. 4 PL spectrum of as-grown GaN nanostructures ammoniated at $950^{\circ} \mathrm{C}$.

According to the above analysis, as for the growth mechanism of synthesis of the GaN nanostructured films, we might briefly explain as follows: On one hand, $\mathrm{ZnO}$ film is initially fabricated on $\mathrm{Si}$ substrate by sputtering $\mathrm{ZnO}$ target through radio frequency magnetron sputtering system. Then the sample is annealed in oxygen ambient at the temperature of $700^{\circ} \mathrm{C}$. A better crystal structure and more relax $\mathrm{ZnO}$ film is achieved, which is much better than the sputtering $\mathrm{ZnO}$ film, but slightly worse than that of $\mathrm{ZnO}$ films annealed at $900^{\circ} \mathrm{C}[18]$. The $\mathrm{ZnO}$ films with a slight roughness bring some defects for the subsequent growth of GaN film, which presumably assists the growth of GaN nanostructures. On the other hand, when the temperature is above $650^{\circ} \mathrm{C}$, the $\mathrm{ZnO}$ films annealed in $\mathrm{NH}_{3}$ ambient will evaporate. The volatilization process may conduct the following reaction:

$$
\mathrm{ZnO}+\mathrm{NH}_{3} \rightarrow \mathrm{Zn}+\mathrm{NO}_{2}(\text { or } \mathrm{NO})+\mathrm{H}_{2} \mathrm{O}
$$

$\mathrm{ZnO}$ film reacted with $\mathrm{NH}_{3}$ to produce $\mathrm{Zn}, \mathrm{NO}_{2}$ (or $\mathrm{NO}$ ), and water vapor. $\mathrm{Zn}$ sublimed at the high temperature and was brought away by $\mathrm{NH}_{3}$ gas to the inner wall in the downstream of the tube [21]. It explains why the $\mathrm{ZnO}$ buffer layer has completely volatilized at $950^{\circ} \mathrm{C}$. The volatilization of $\mathrm{ZnO}$ buffer layer plays an important role in the formation process of $\mathrm{GaN}$ nanostructures. Although $\mathrm{ZnO}$ buffer layer has completely evaporated during the annealing process, it could improve nucleation by lowering the surface energy and providing an improved lattice match in the growth of GaN film. It is unavoidable to form some defects and dislocations, which can subsequently be used as a mask or growth sites to fabricate the nanorods or act as potential nucleation sites for the GaN nanostructures, and result in formation of $\mathrm{GaN}$ crystal nuclei at the above-mentioned sites. When one $\mathrm{ZnO}$ layer volatilizes, the amorphous $\mathrm{GaN}$ layer falls on the next $\mathrm{ZnO}$ layer so as to decrease the interfacial energy. At the end, the GaN nanostructured film will fall on the Si substrates directly. Then the very small GaN granules grow up with the progress of the ammoniating process and accordingly lay the foundations for the growth of nanostructured $\mathrm{GaN}$. These GaN molecules continuously come out and agglomerate into micrograins. When the growth direction of the micrograins orientate in the same direction, the single $\mathrm{GaN}$ nanorods are formed. Under these conditions, amorphous $\mathrm{GaN}$ films reorganize and become GaN nanostructured films: nanorods, nanopaticles and nanotubes. 


\section{Conclusions}

$\mathrm{GaN}$ nanostructured films are fabricated on $\mathrm{Si}(111)$ substrates with $\mathrm{ZnO}$ buffer layer by radio frequency magnetron sputtering system. Then the as-grown films are annealed at $950^{\circ} \mathrm{C}$ in $\mathrm{NH}_{3}$ ambient. The results show that the nanostructers are pure hexagonal $\mathrm{GaN}$ wurtzite structure. The representative photoluminescence spectrum at room temperature exhibited a strong emission peak at $374.1 \mathrm{~nm}$ and two weak emission peaks at $435.9 \mathrm{~nm}$ and $474.8 \mathrm{~nm}$. Finally, the proposed growth mechanism is also briefly discussed.

\section{Acknowledgements}

This work was supported by the National Natural Science Foundation of China (11404178).

\section{References}

[1] M.J. Majid, A.B. Rodrigo, K. Irma, P. Victor and D.E. Horacio: Nano Lett. Vol. 12 (2012), p. 970

[2] L.T. Fu, Z.G. Chen, D.W. Wang, L. Cheng, H.Y. Xu, J.Z. Liu, H.T. Cong, G.Q. Lu and J.Zou: J. Phys. Chem. C Vol. 114 (2010), p. 9627

[3] R. Yu, W.Z. Wu, Y. Ding and Z.L. Wang: ACS Nano Vol. 7 (2013), p. 6403

[4] K.S. Boutros, S. Chandrasekaran, W.B. Luo, V. Mehrotr, in: Proceedings of the 2006 IEEE International Symposium on Power Semiconductor Devices and IC's (2006) p. 1

[5] K. Lekhal, Y. André, A. Trassoudaine, E. Gil, G. Avi, J. Cellier and D. Castelluci: Cryst. Growth Des. Vol. 12(2012), p. 2251

[6] S. Dai, J. Zhao, M. He, X.G. Wang, J.C. Wan, Z.W. Shan and J. Zhu: Nano Lett. Vol. 15 (2015), p. 8

[7] J. Heo, S. Jahangir, B. Xiao and P. Bhattacharya: Nano Lett. Vol.13 (2013), p. 2376

[8] D. Li, M. Sumiya, S. Fuke, D. Yang, D. Que, Y. Suzuki and Y. Fukuda: J. Appl. Phys. Vol. 90 (2001), p. 4219

[9] Y. Sun, T. Miyasato and J.K. Wigmore: J. Appl. Phys. Vol. 85 (1999), p. 3377

[10] F.M. Amanullah, K.J. Pratap and V.H. Babu: Mater. Sci. Eng. B Vol.52 (1998), p. 93

[11] P. Perlin, C. Jauberthie-Carillon, J.P. Itie, A. San Miguel, I. Grzegory and A. Polian: Phys. Rev B Vol. 45(1992), p. 83

[12] S. Xue, H. Zhuang, C. Xue, L. Hu, B. Li and S Zhang: Appl. Phys. A Vol.-87 (2007), p. 645

[13] Y. Dikme, G. Gerstenbrandt, A. Alam, H. Kalisch, A. Szymakowski, M. Fieger, R.H. Jansen and M. Heuken: J. Cryst. Growth Vol.248 (2003), p. 578

[14] J.R. Grandusky, V. Jindal, N. Tripathi, F. Shahedipour-Sandvik, H. Lu, E.B. Kaminsky and Melkote: J Cryst. Growth Vol.307 (2007), p. 309

[15] X. Mao, Z.Yang, J. Li, J. Qu and G. Zhang: Chinese J Semiconductors Vol.20 (1999), p. 639

[16] Y. Sun, T. Miyasato and N. Sonoda: J. Appl. Phys. Vol.84 (1998), p. 6451

[17] X. Chen, J. Li, Y. Cao, Y. Lan, H. Li, M. He, C. Wang, Z. Zhang and Z. Qiao: Adv. Mater. Vol. 12 (2000), p. 1432

[18] S. Xue, H. Zhuang, C. Xue, L. Hu, B. Li and S. Zhang: J Electron Mater. Vol. 36 (2007), p. 502 